\title{
VIRTUAL REALITY AND ITS APPLICATIONS IN CHINA: AN OVERVIEW
}

\author{
Zhigeng Pan \\ The Hong Kong Polytechnic University, Hong Kong \\ Zhejiang University, P.R. China \\ Jiaoying Shi \\ The Hong Kong Polytechnic University, Hong Kong \\ Qin Lu \\ Zhejiang University, P.R. China
}

\begin{abstract}
Nowadays Virtual Reality (VR) has become a hot research topic. In China researchers have obtained a lot of achievements in various VR fields. VR is no longer limited to academic research as it used to be, it has sprung up to a new industry. This paper gives an overview of the research and development on VR in China, including the mainland of China, Hong Kong and Macao. It also gives a summary of the 11 selected papers in the printed edition of this and the next special issues.
\end{abstract}

\section{Introduction}

The research work in computer graphics (CG), started in the 1960s, became a well-established research field in the 1980's [1]. As the research work has become more mature, commercial CG related hardware systems and software systems have appeared in wide use and the industry has become well established. CG education and research are now being conducted at universities and institutes, as well as in industrial training centers [2,3].

As a new branch of CG, virtual reality (VR) has attracted a lot of attention in recent years. VR is the use of computer graphics systems in combination with various display and interface devices to provide the effect of immersion in the interactive $3 \mathrm{D}$ computer-generated environment. We call such an environment a virtual environment (VE). Research and development of VR and VE applications can be found in many places all over the world. In China, the research and development work related to VR began in the early 1990s. In less than 10 years researchers in China have produced quite a number of significant research results in various VR fields due to the strong support for fundamental research from the National Science Foundation of China and the National Key Programs of Science and Technology. Relevant industrial sectors have also given very strong support to applied VR research [4].

The remainder of this paper is organized as follows. Section 2, 3, and 4 introduce VR research and development in various universities, institutes, and commercial companies respectively. Section 5 gives a brief overview of the international conferences and workshops related to VR held in China. Section 6 summarizes selected papers in the printed edition of this special issue. Finally, Section 7 gives closing comments. 


\section{VR Research in Universities}

\subsection{ZHEJIANG UNIVERSITY}

As the largest university in China, Zhejiang University is composed of five campuses in different locations in Hangzhou. Three labs largely focus on research work in various VR fields.

\section{State Key Lab of CAD\&CG}

This lab is one of the first units in China that started the research work on VR at the beginning of 1990s. The lab received funds for eight VR-related projects from the National Science Foundation of China in the past few years. It should be noted that the lab has established the first CAVE system in China in June 1999 (see Fig. 1).

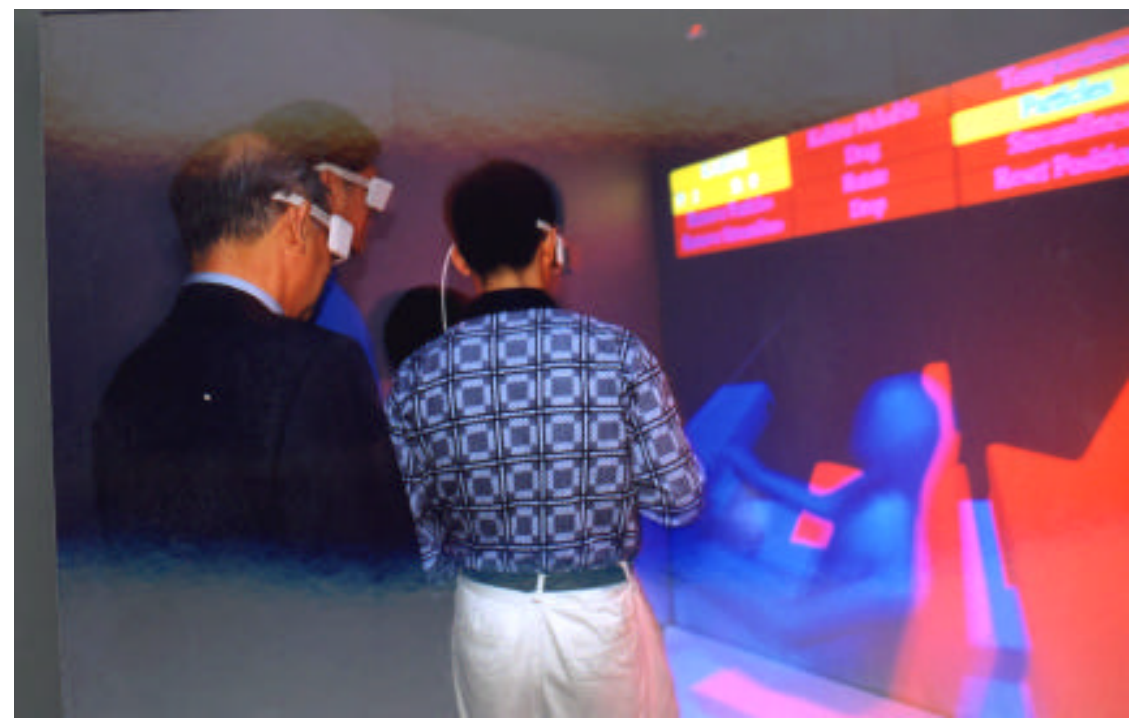

Fig. 1 The first CAVE system established in China

In Level of Detail (LoD)/multi-resolution direction, several new mesh simplification algorithms were proposed [5], including a fast generation algorithm for progressive meshes [6], a multiresolution modeling system, and new multi-resolution BSP tree [7]. (Fig. 2 shows a LoD example created with our algorithm). Static LoD and dynamic LoD methods are both explored. A new fast rendering algorithm incorporating both kinds has been developed. First, the polygonal models are simplified based on a user-specified error approximation, which is independent of viewpoint. Then the simplified models are used in a view-dependent real-time rendering algorithm. 


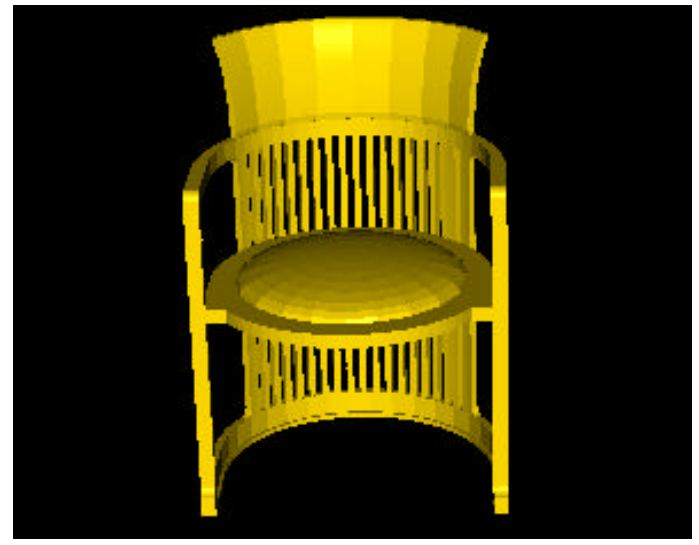

(a) 3760 triangles

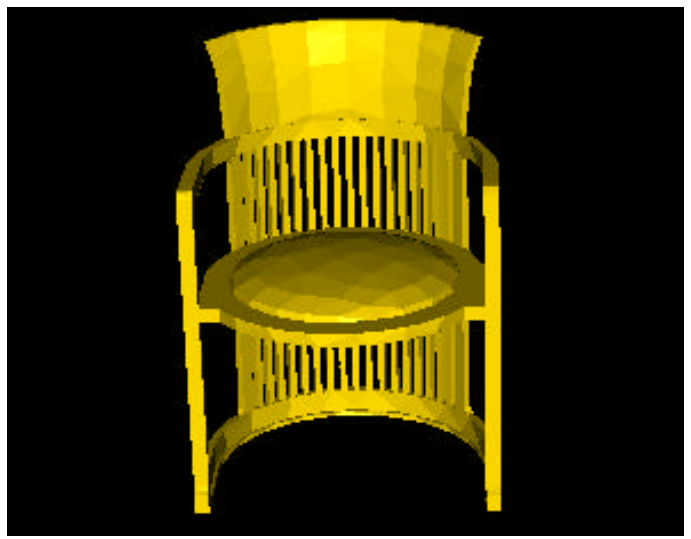

(C) 734 triangles

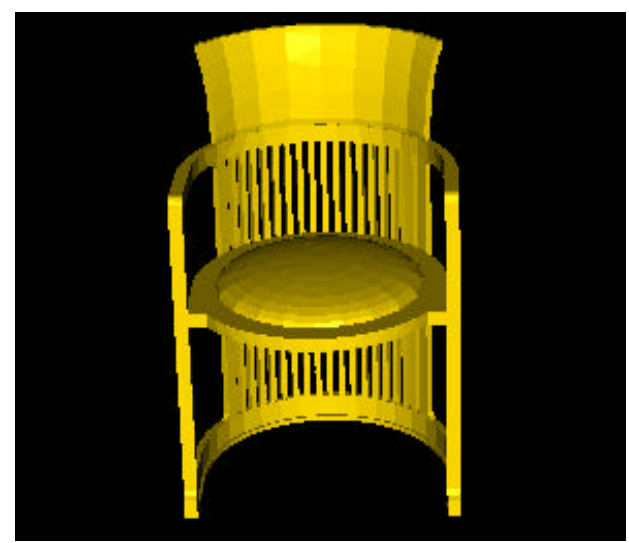

(b) 1484 triangles

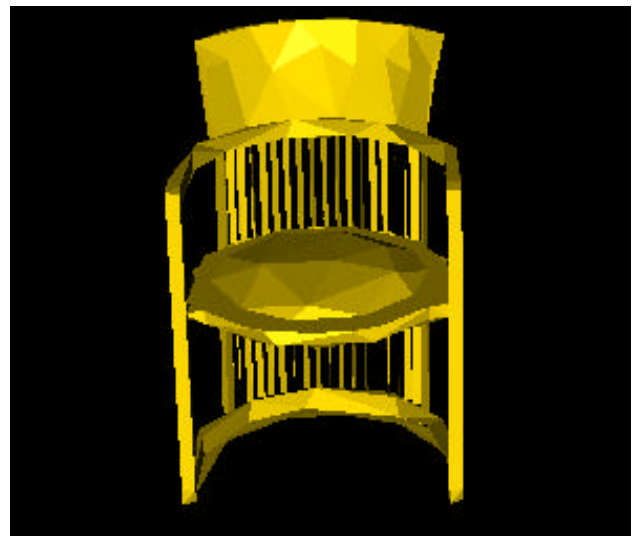

(d) 323 triangles

Fig. 2: A LoD example created with our algorithm

In real-time rendering, research works mainly focus on visibility pre-computing technique [8,9], nonlinear view interpolation technique [10], and geometry and image based hybrid-rendering technique. The visibility pre-computing algorithm samples the environment in a $3 \mathrm{D}$ lattice. The potentially visible surfaces for each node are computed and stored, and the distant visible surfaces are simplified as textures. During walkthrough in a node, only the potentially visible surfaces and the textures attached to the node need to be rendered. The nonlinear view interpolation algorithm simulates precisely the perspective transformation during walkthrough. A binary decomposition scheme is proposed to reduce the number of sub-image blocks, which greatly improves the efficiency of the traditional view interpolation.

In addition, some other research results have been obtained in the area of image-based modeling and rendering (IBMR) based on wavelets [11,12], distributed virtual reality [13], time-critical rendering [14], and 3D acoustic rendering for virtual environments (VE) [15]. The Virtual ZJU (Virtual Zhejiang University) is a virtual touring system, which was implemented, based on IBMR. Fig. 3 is a snapshot of the images in this virtual touring system showing the gate of Yuquan Division, Zhejiang University. Some VR applications (medical treatment and surgery planning, architecture walkthroughs, and $\mathrm{CAD} / \mathrm{CAM}$ ) are being developed using the CAVE environment. 


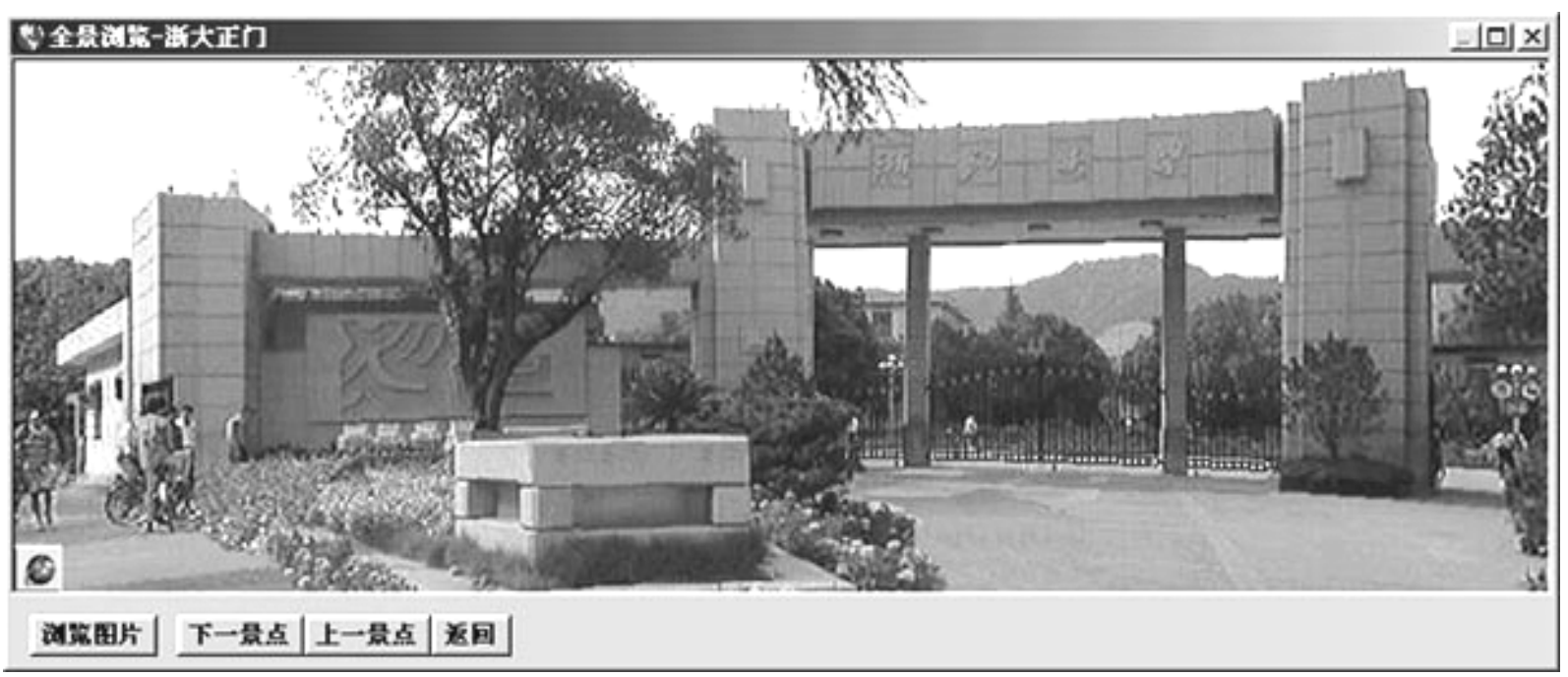

Fig. 3: A snapshot in the Virtual ZJU system Intelligent CAD Lab, Department of Computer Science and Engineering, Zhejiang University

VR research in this lab focuses on the IBMR method and its application. A project called Virtual Dunhuang Art Cave (DAC) was developed as a virtual navigation system [16]. The system provides new techniques and facilities for DAC presentation, investigation, and exploration. A demo view from navigation is shown in Fig. 4. Another VR application is the VR TOURISM system [17], which uses panoramas and picture-based animation to compose a virtual touring environment.
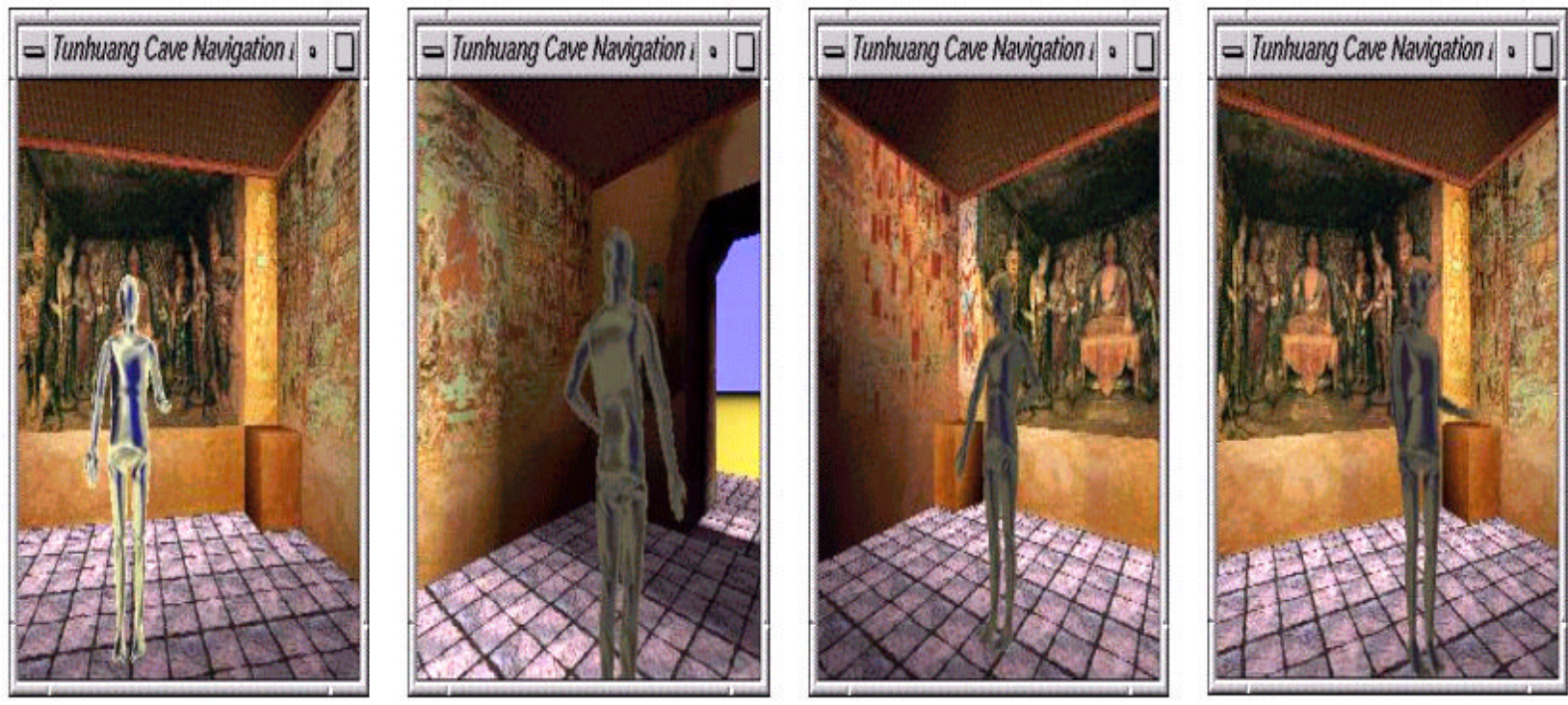

Fig. 4: Demo view from navigation of Virtual DAC National Lab of Human Factors, Zhejiang University

This lab, which belongs to the former Hangzhou University, developed a VE called a Virtual Forbidden City based on geometric modeling [18]. The first version was implemented on SGI machine(s) with the MultiGen tool. Later it was ported to the PC platform in cooperation with Intel Corp. Users can now also view the scenes of Forbidden City on the Internet. Recently the environment is extended to a distributed VE (Fig. 5 is an example). 


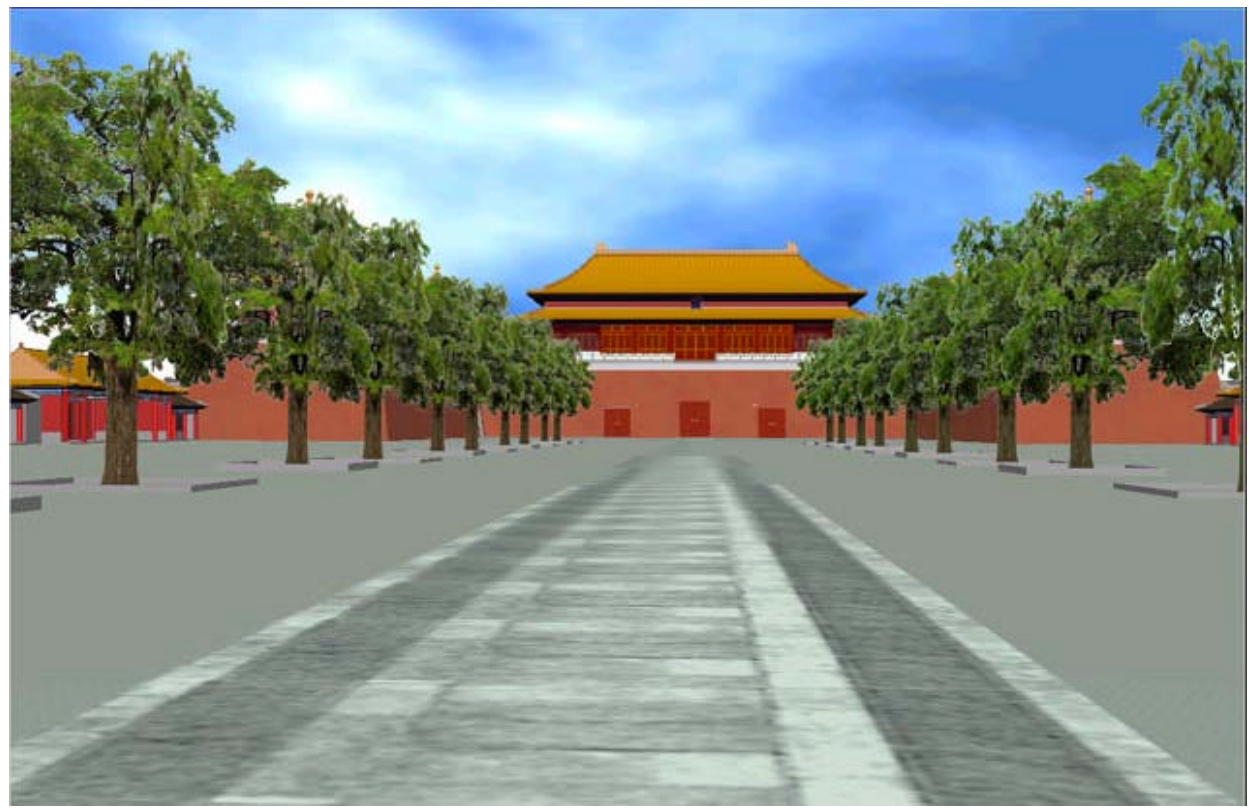

Fig.5: A snapshot in the virtual Forbidden City

\subsection{DEPARTMENT OF COMPUTER SCIENCE AND TECHNOLOGY, TSINGHUA UNIVERSITY}

VR research in Tsinghua University covers not only continuous LoD models, multi-resolution modeling, and real-time rendering [19,20], but also augmented VR. Research results include a new hierarchical multi-resolution modeling method based on fractal dimension, a real-time continuous LoD rendering method for 3D complex models, and modified mesh simplification algorithms. VR techniques are applied to neuro-surgery planning by the technicians in this department in cooperation with the Navy Central Hospital in Beijing. Another important research subject is in Augmented VR. Researchers used a novel approach to construct 3D real full-view model by dithering video sequences [21]. In addition, a VR development platform and a VR system for dental surgery planning and simulation have been implemented.

\subsection{NATIONAL UNIVERSITY OF} DEFENCE TECHNOLOGY

Research work in the National University of Defense Technology includes distributed virtual reality [22], behavior modeling [23], IBMR tool [24], simulation of dynamic ocean waves, collision detection, and applications of multi-agent methods to VE [25]. YHVRP is a prototype of a DVE application development system. It provides developers with a modeling language called $Y H V M L$, having a language model of VE based on complex behavior. YHVML also supports 3D geometry modeling. Another project, $H V S$, is a VR authoring tool based on IBMR. It supports the display of up to 800 by 600 resolution with photo-quality effects. The creation of virtual environments is made simple by getting rid of the complex 3D modeling process all together.

\subsection{BEIJING UNIVERSITY OF AERONAUTICS AND ASTRONAUTICS}

A distributed VE system developed at this University, referred to as DVENET (distributed virtual environment network) [26] supports dynamic simulation of flight using object behavior description [27] and object-oriented collision detection [28]. DVEMET includes a wide-area network, a set of standards and toolkits, as well as other resources such as 3D terrain, all of which can be used in a 
distributed virtual environment in a wide range of fields. In addition, based on DVENET, a distributed virtual battlefield environment has been developed. In an exercise, hundreds of entities controlled by different nodes, which are distributed from different locations, can take part in and interact in a $110 \mathrm{~km}$ by $150 \mathrm{~km}$ synthetic battlefield to support joint exercises of a variety of forces.

\subsection{COMPUTER GRAPHICS GROUP OF THE UNIVERSITY OF HONG KONG}

The Computer Graphics Group of the University of Hong Kong started teaching and doing research in VR in 1995. At the early stage the main effort was to study fundamental issues of virtual environments, such as collision detection and visibility computation [29,30]. These results, plus some other speedup techniques, were later integrated into the first VR walkthrough system of the group, which models the two floors of a building that houses department offices and laboratories [31]. Currently the group is actively engaged in several interdisciplinary VR-related applications, including virtual orthopedic surgery planning and flow visualization for environmental assessment in civil engineering.

\subsection{VR, VISUALIZATION, AND IMAGING RESEARCH CENTRE AT THE CHINESE UNIVERSITY OF HONG KONG}

This is the first government funded VR research center in Hong Kong, focusing on the development of practical virtual environments for medical and engineering applications. One pilot project of this center is to develop an intelligent virtual environment for computer simulation of bronchoscopy, a medical procedure used to diagnose and treat lung diseases [32].

The researchers at this center have worked on virtual hand interfaces using fuzzy logic and physics-based augmented hand interactions, as well as its applications in robotics simulation, engineering assembly, and virtual manufacturing [33]. Other work in the area of image-based VR is also conducted in order to facilitate interactive illumination and navigation control in an imagebased environment [34]. Fig. 6 is an image generated by the warping method. In addition, VR techniques have been used in architecture applications for the Department of Architecture (See Fig. 7).

\subsection{VR LAB OF THE HONG KONG POLYTECHNIC UNIVERSITY}

At the VR lab in the Department of Computing, researchers developed a progressive view morphing method [35] and wavelet-based image mosaics using edge-preserving visual perception modeling [36]. They have also applied multiresolution modeling to virtual design and prototyping [37]. Currently the VR Lab is planning a 4-sided CAVE based on the SGI ONYX machine, the first of its kind in Hong Kong. CAVE was the first virtual reality technology in the world to allow multiple users to fully immerse themselves simultaneously in the same virtual environment. It is one of the most exciting VR output devices available today. In addition, VR technology is being applied to help the visual impaired. Other VR research work includes Web-based virtual environments, virtual environments for furniture design and layout, and collaborative virtual environments. 

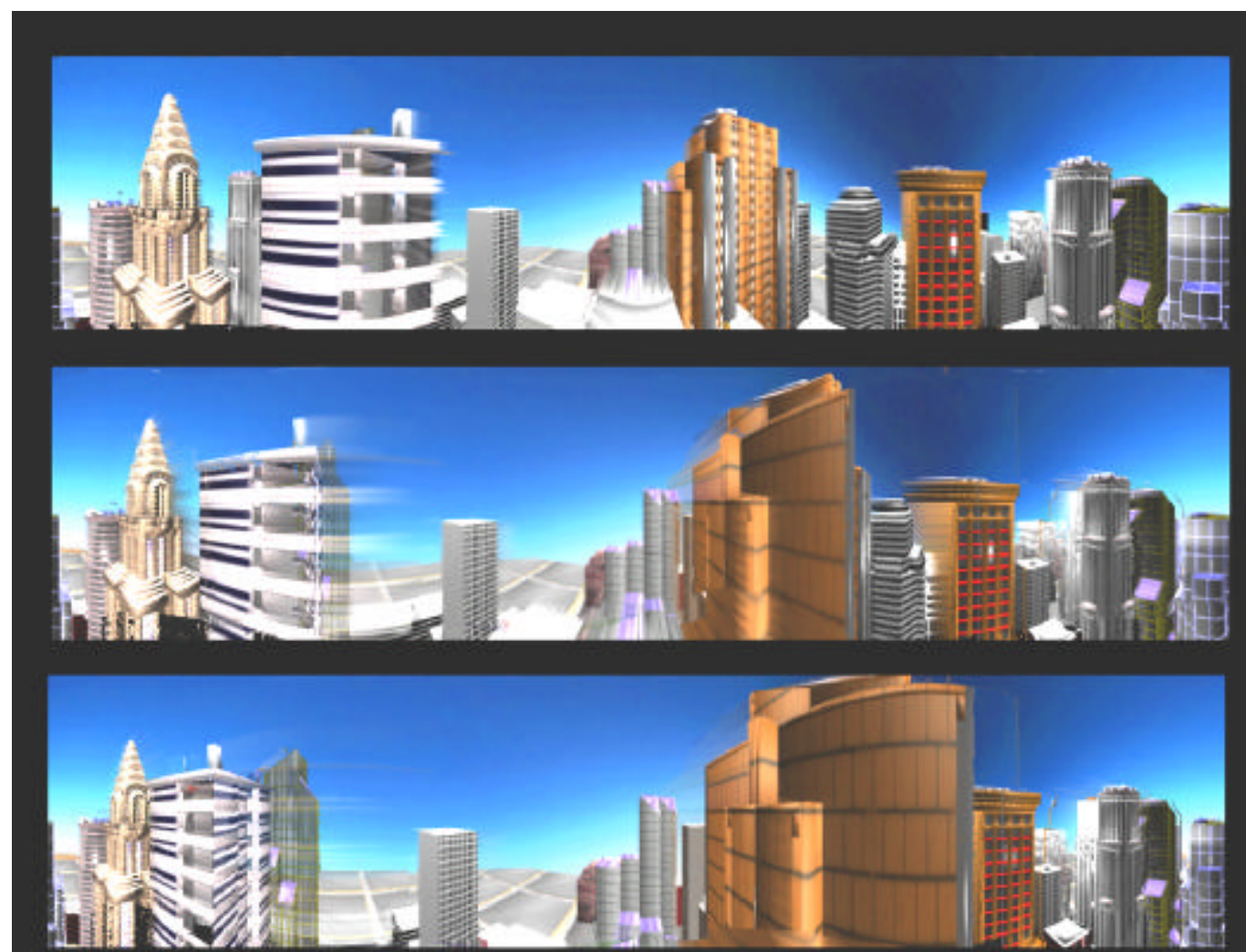

Fig. 6: Warpcity image

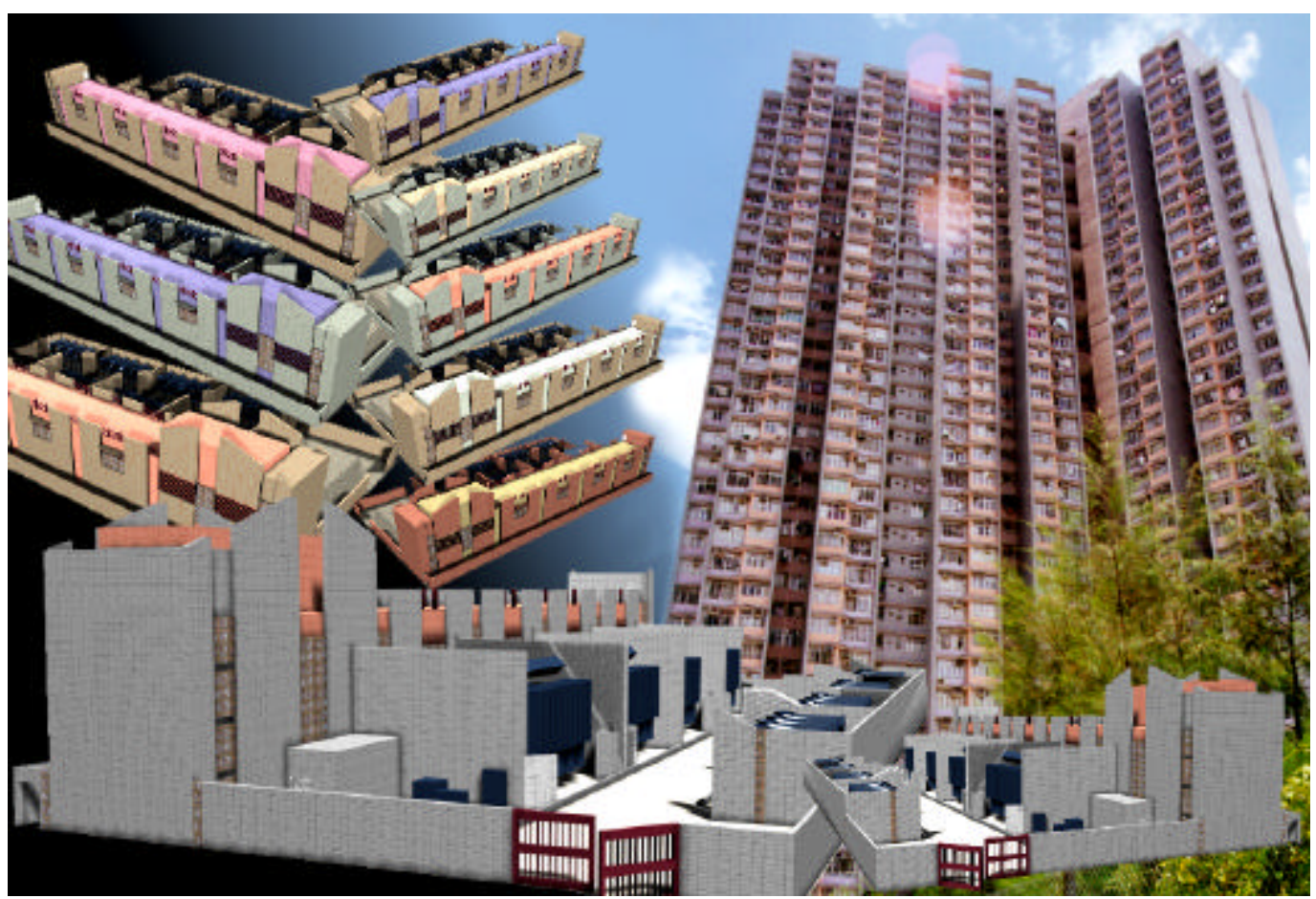

Fig. 7: Architectural group 
The VR research of this group focuses on three major projects in the VR area. The first is to develop efficient a multiresolution technique [38] for object modeling (see Fig. 8), in particular, techniques that would take into account the dynamically changing factors. The second is to develop an efficient method for handling deforming surfaces. Both of the projects aim to improve the rendering performance in time-critical applications. The third is to develop a distributed VR system for a walkthrough of large virtual environments over the Internet $[39,40]$. Instead of transmitting the complete geometry database to the client before the start of the walkthrough, as most systems do, this system is based on the on-demand transmission of object models to the client in the form of progressive meshes.

\subsection{OTHER UNIVERSITIES}

Some other important research results have been achieved at other universities. They include a PC-based VR platform from Beijing University [41], a virtual simulator for the training of automobile driving, as well as a computing model for VR [42,43] from Nanjing University (See Fig. 9), stereo graphics generation and stereo TV in Xian Jiaotong University, a virtual walkthrough system for architectural VR and a continuous multiresolution modeling algorithm from Tianjing University [44], an application development tool at Harbin Institute of Technology [45], a locomotive driving simulator based on MPEG video in Southwest Jiaotong University, VR applications to engineering in Northwest Polytechnic University, a VR-based CAM, focused on the layout of a workshop in a VE at Shanghai Jiaotong University. Some IBR work has been carried out at the Hong Kong University of Science and Technology. In addition, a project on the recreation of a historic relic environment for St Paulo Church in Macao based on its ruins is being conducted at the University of Macao [46] (See Fig. 10).

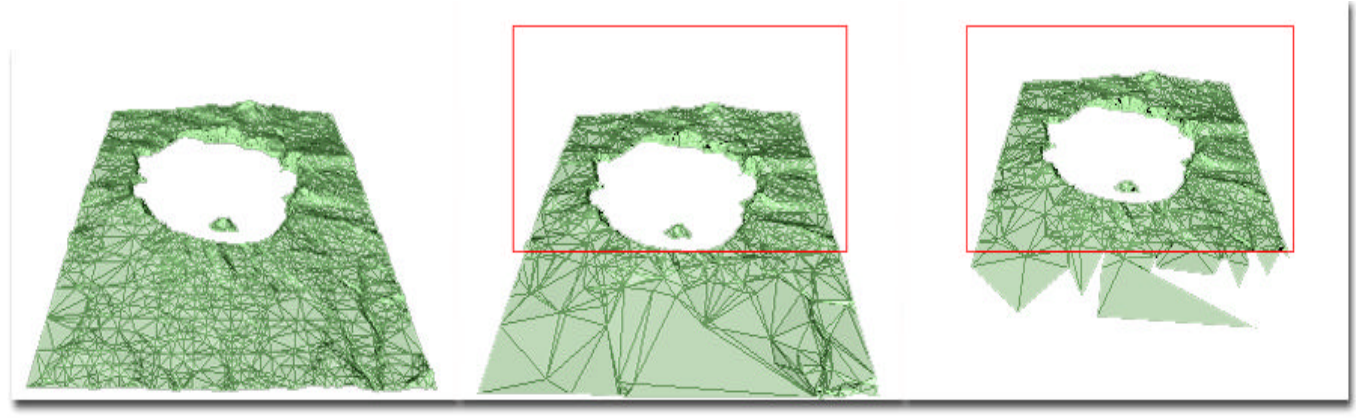

(a) 9,620 triangles

(b) 4, 353 triangles

(c) 2,768 triangles

Fig. 8: The diagram shows the selective transmission and progressive construction of a landscape model. (The rectangular box represents the area of interest.) 


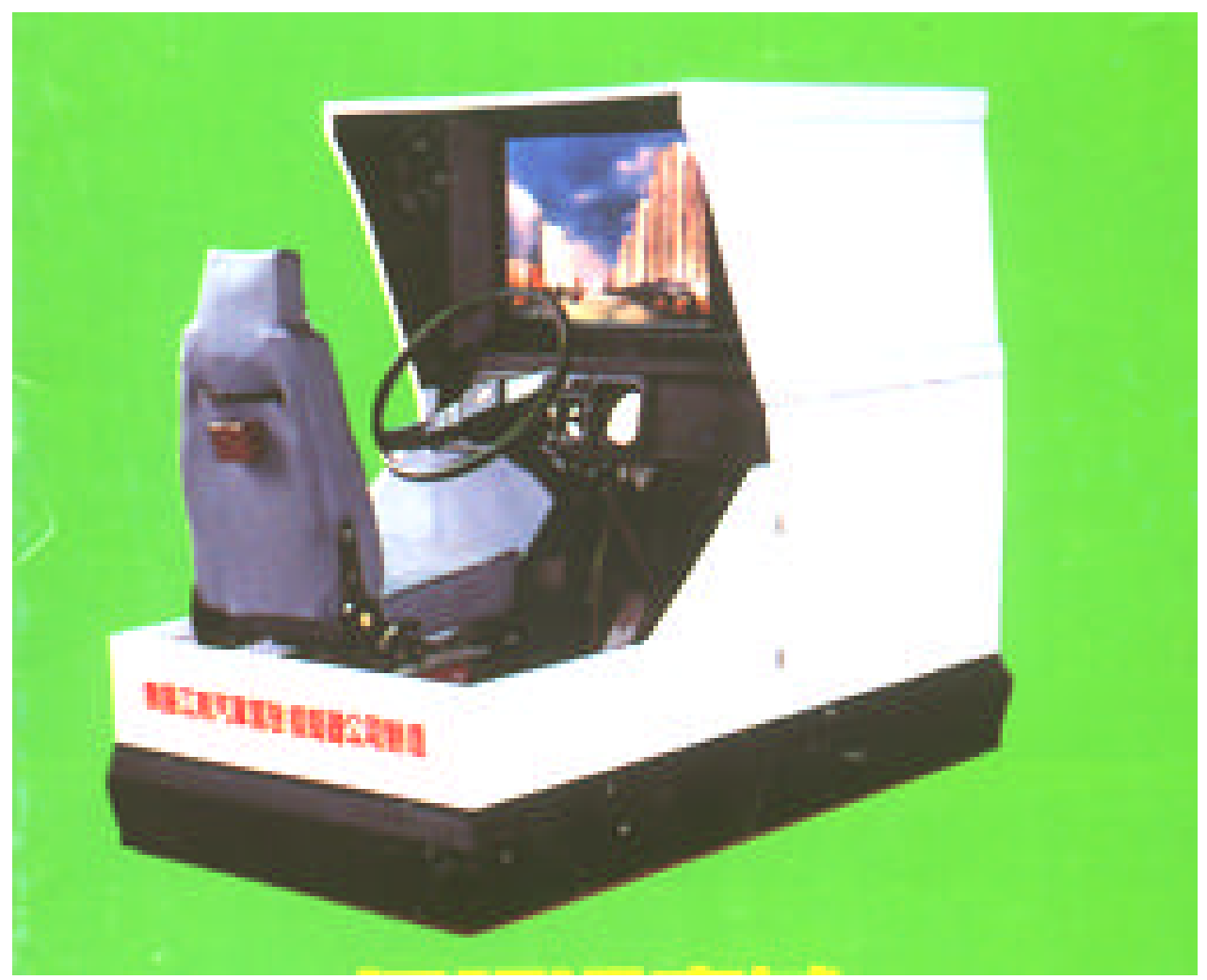

Fig. 9: Driving simulator developed by Nanjing University.

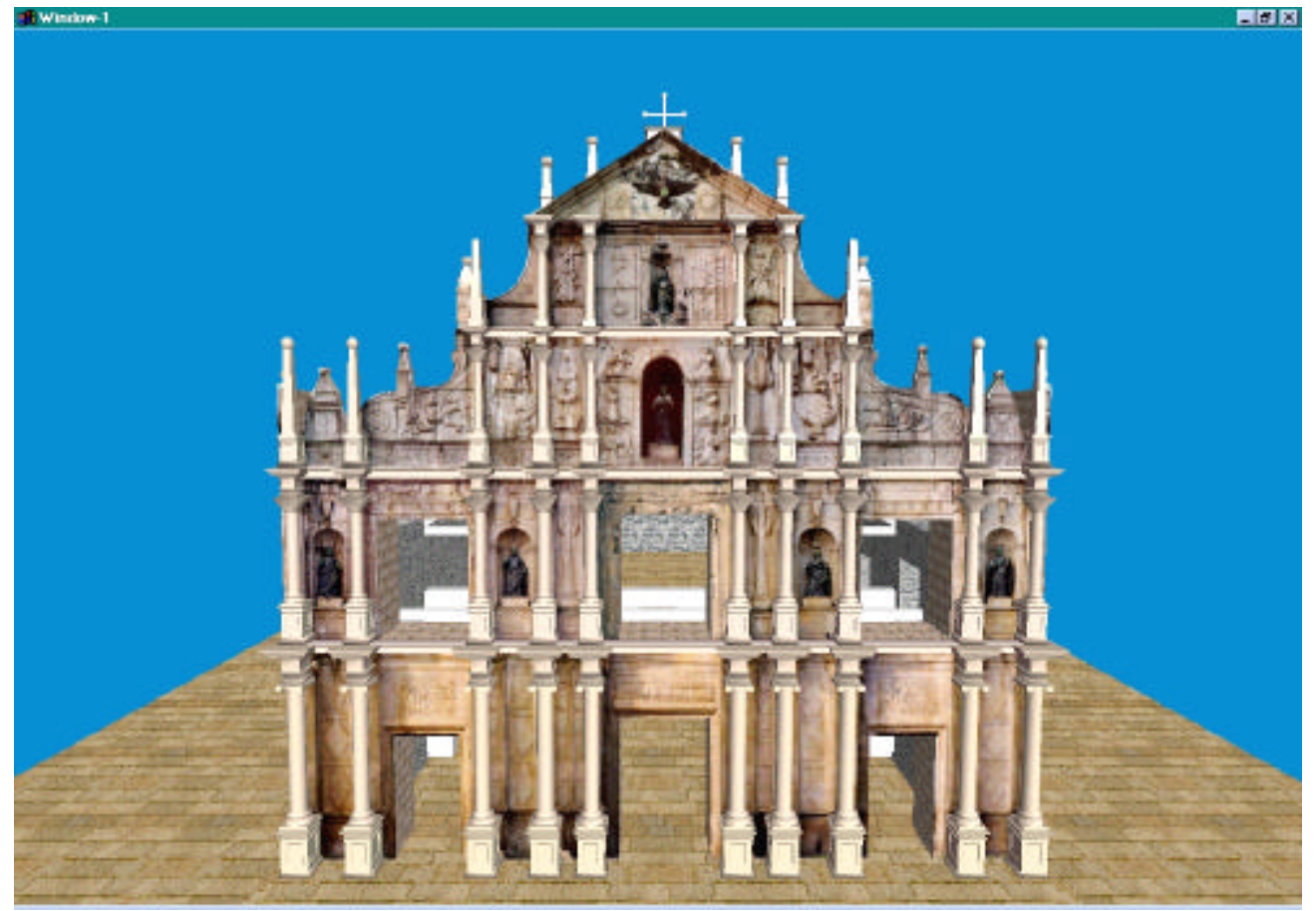

Fig10: Virtual St Paulo Church in Macao 


\section{VR Research at Institutes}

\subsection{CAD LAB OF THE INSTITUTE OF \\ COMPUTING TECHNOLOGY AT THE \\ CHINESE ACADEMY OF SCIENCES}

This lab has conducted quite a number of projects in computer graphics, visualization, and CAD. One is "Dunhuang", a government-funded project dedicated to virtual recovery of endangered culture relics. In order to recover the accurate position of objects and camera from several regular photos, the researchers proposed a new method by solving a so-called "PnP problem." To obtain the on-the-fly effect in a walkthrough of the virtual cave, both IBMR and geometry-based rendering are employed [47]. To deal with large data and the extraction of useful information accurately and quickly, the Haar wavelet transformation has been used to create multiresolution images and indexing through discrete quad-tree. To realize the smooth transition of such images, a fractionallevel zooming algorithm is presented to zoom portions of a multiresolution image to any arbitrary size [48]. In addition, extensive research has been carried out in the area of distributed virtual reality, animation [49], and natural texture generation.

\subsection{LAB OF COMPUTER SCIENCE AT THE INSTITUTE OF SOFTWARE, CHINESE ACADEMY OF SCIENCES}

This lab has recently been engaged in the research and system implementation on virtual reality. The research is mainly focused on the subjects of $\mathrm{LoD}$ [50], image based rendering, hybrid rendering of images and graphics, real-time rendering techniques including visibility processing, and real-time LoD techniques [51]. For VR system design and implementation an image-based VR system called QuickShow [52] was implemented based on the approach of panoramic view composition using a cylinder surface. A prototype VR teaching environment was also established using graphical models. The environment covers conventional teaching facilities including classrooms, laboratories, and so on (See Fig. 11).

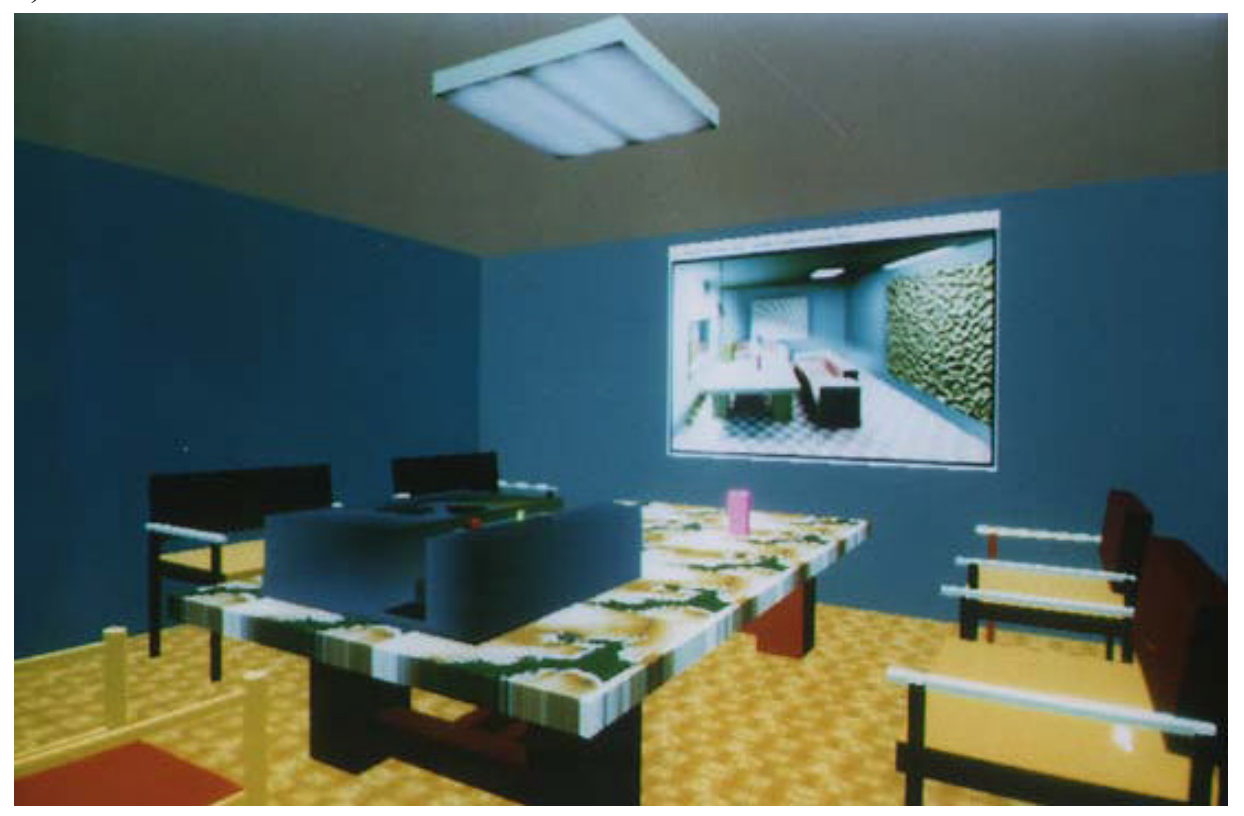

Fig. 11: Virtual classroom 


\subsection{LAB OF ENGINEERING SCIENCE FOR COMPLEX SYSTEMS, INSTITUTE OF AUTOMATION AT THE CHINESE ACADEMY OF SCIENCES}

Some research work on a new kind of flexible angle sensor has been done in this lab, and a kind of dataglove called IA_Glove has been developed using the sensor. The staff there is also developing a new method for designing and simulating the product line with robotic work-cells. Other research results include mesh simplification, multi-level collision detection.

\section{VR Developments in Industry}

\subsection{SUNSTEP INC., BEIJING}

SunStep Inc. develops, manufactures, and markets the AGC line of very high-end graphics products. Their products are used worldwide in many real-time and time-critical applications, such as air traffic control, flight simulation, electronic mapping, CAD, and VR.

The $A G C-2075+$ is evaluated as the best graphics card by $P C$ Computing magazine published in USA. The $A G C-400$ graphics card can support high-resolution of 2048 by 2048 , while the $A G C-G L$ 300 is the first real-time 3D graphics accelerator developed in China. The AGC-ProV is the first VRoriented stereo display card in the world. It employs hardware implementation of stereo display with high-resolution, high stability, and high field frequency up to $160 \mathrm{MHz}$. Furthermore, it has three hardware accelerating engines (100\% OpenGL acceleration, $100 \%$ texture acceleration, and geometric operation). It can support all high-end application software that needs OpenGL. There are two types of products for AGC-VR series stereo display systems: AGC-ProV1 and AGC-ProV2. They are composed of a stereo display card, stereo glasses with or without wire, emitters, and a stereo software development tool. The company has also developed a new low-cost data glove called the AGC-DataGlove, which will be on the market very soon.

\subsection{SHANGHAI 3D TECHNOLOGY} CO., LTD, SHANGHAI, CHINA

This company is a subsidiary of 3D Inc in Japan, which develops applications and provides products on simulation, visualization, and VR flight simulation (See Fig. 12), weather broadcasting, a virtual shopping mall, data visualization, etc). Now the company is focusing on a haptic rendering technique, which may be used in medical surgical training system and clay modeling. Applications are also being developed for surgery planning and other specific fields.

Currently this company has the following VR-related products. (a) $3 D++/$ Weather, for weather forecasting with 3D images; (b) GeoMagic polygon reduction software using Wavelet technology; and (c) $V s \_l i b$, a library to support the development of CAVE applications. Vs_lib runs on the SGI platform. There are two ways to write applications for the VS system: "vs level" is used for highlevel programming. "xx level" contains the lower level functions for devices support and system setup. 

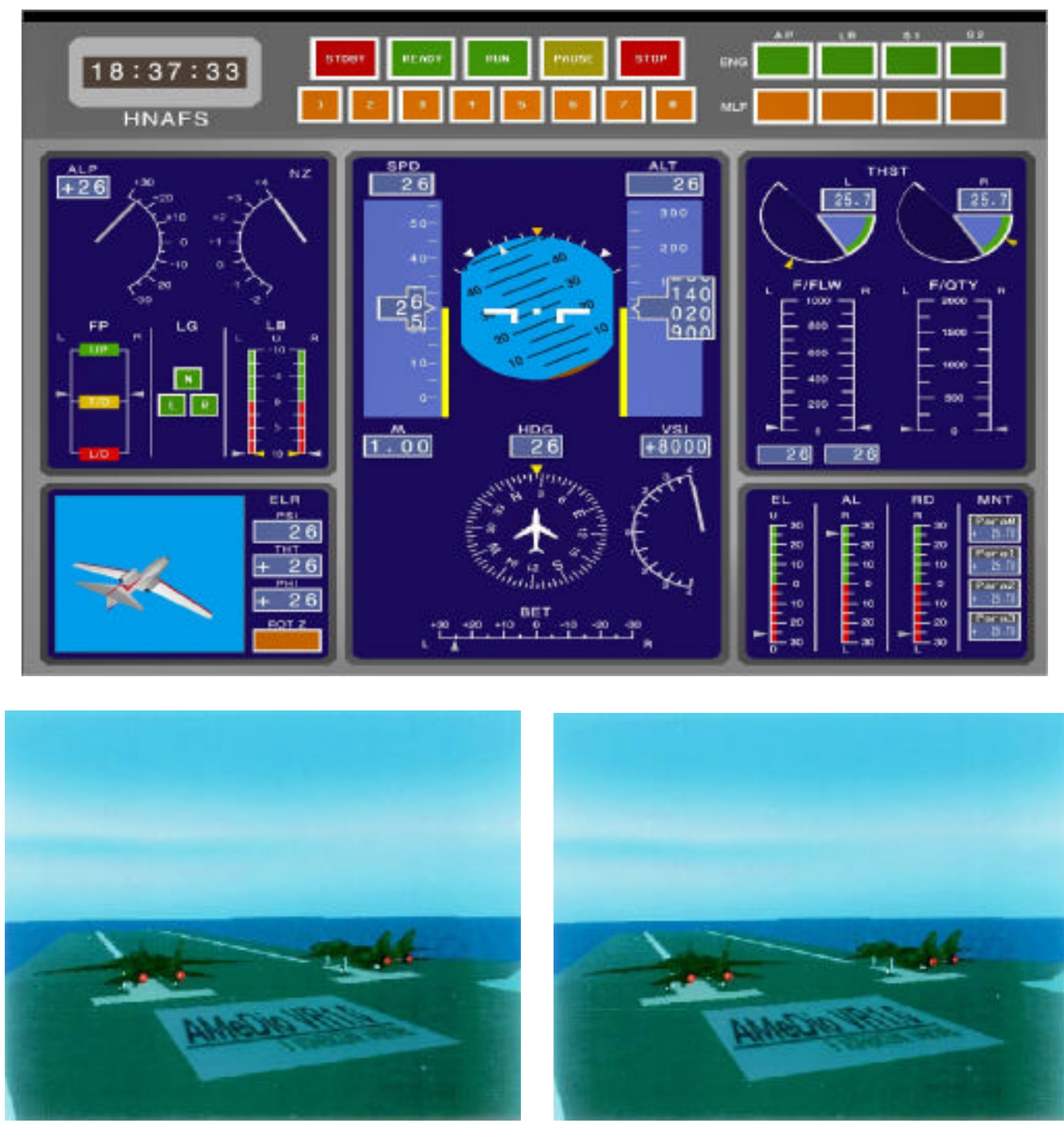

Fig. 12: Snapshot of Flight simulation system

\subsection{VOYAGER CORP., ZHONGSHAN, GUANGDONG PROVINCE}

Reality Builder 2.0, produced by the Voyage Corp., is a 3D interactive scenario generation tool that runs on IBM compatible PCs. The product was developed in MS C++ 4.0 developer studio, a 32-bit API. The programming languages supported are Visual $\mathrm{C}++$ and $\mathrm{C}$. MFC and Win32 APIs are used as the underlying support environment.

Image Generator (Eagle) Network Platform is another software product that supports network image generators for interactive games. Each station may have a single or multiple image generator (with wide field-of-view) options. The number of stations depends on the capability of the server. The maximum capacity is 31 stations.

Besides software products, this company also has hardware products for VR application. One typical example is the so-called Tiger IG ("IG" stands for "image generator"), special hardware used for 3D real-time image generation. It employs an IBM compatible PC as the host and utilizes a PCI slot as the interfacing port. Tiger IG utilizes BSP (binary separation plane) to enhance the popular Z- 
buffer approach for hidden surfaces removal. This significantly reduces the bottleneck during the real-time pixel filling process. It provides polygon throughput for up to 1 million four-sided facets per second without pixel size restriction. It also provides pixel-filling rate up to 135 megapixels per second. Therefore Tiger IG is ideal for visual simulation, vehicle driver training, and high-end arcade system applications. Tiger IG is suitable for use as the visual generation core for flight simulation, ship navigation, and vehicle training. It also has vast applications in architecture, transportation planning, CAI, virtual reality, and animation domains.

\subsection{REAL-TIME GRAPHICS INC., CHINA}

This is a software company aimed at real-time simulation. Currently the company provides two products: RTG and EasyVRML. RTG is a toolkit for visual simulation, virtual reality, 3D games, and other real-time 3D graphics applications. The principal design goal is to allow application developers and users to build high performance products and applications easily. To this end, the toolkit combines an RTG scene editor and an RTG engine, supporting collision detection, terrain generation, and many 3D game features. Fig. 13 is an image generated by a VR application based on RTG.

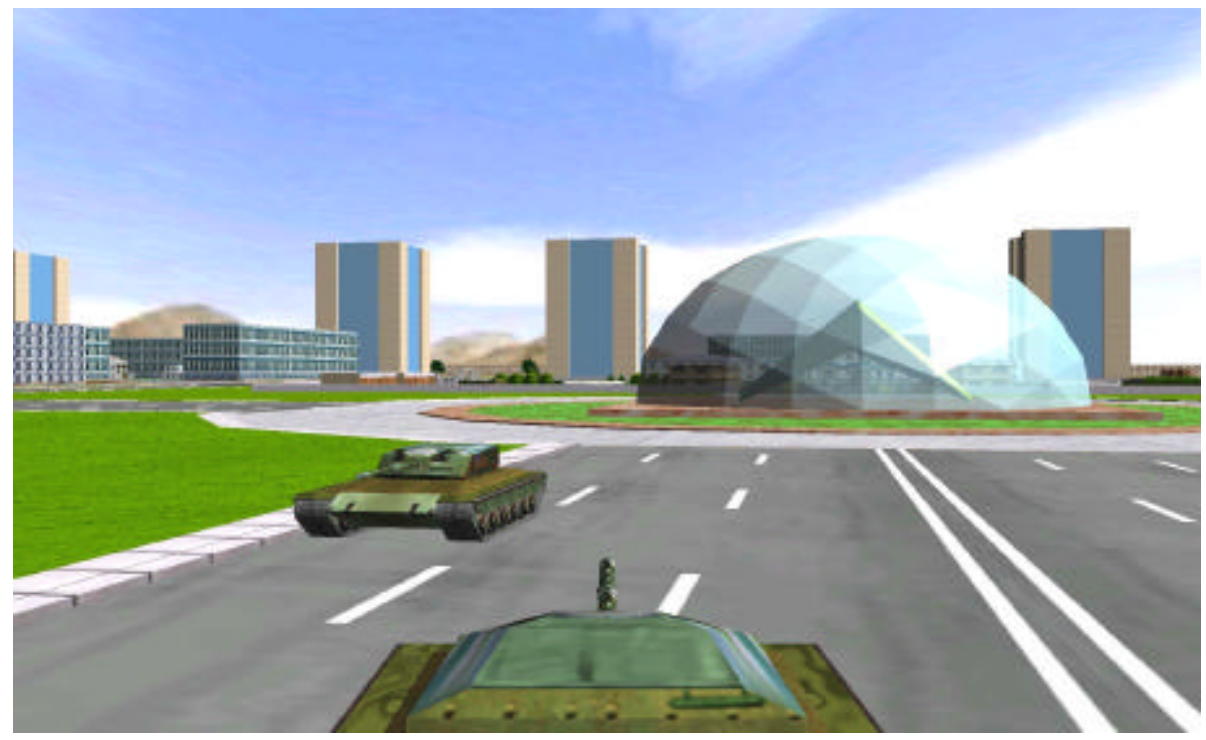

Fig. 13: An image generated by the RTG tool.

EasyVRML is a graphics editor for generating VRML 2.0 code, a format used by the Internet 3D browser. It also includes an ActiveX control for displaying dynamic scenes in PowerPoint, Word, et. al.

\subsection{OTHER COMPANIES RELATED TO VR}

WeiAER Corp. in Shenzhen was established with the cooperation of HK Hongyi Corp. and the Weihai Division of the Chinese Science and Technology Development Institute. It aims at developing practical VR applications and VR-related products. One of its products is the wireless stereo video system, which is composed of 3D stereo image generation component, 3D sound synthesis component, and a stereo interaction control component. It can be used in teaching, games, and design. 


\section{VR Conferences}

Three professional conferences and workshops related to VR were held in China during the past five years. A brief introduction of each is given below.

In 1995 the first international workshop on virtual reality and visualization in scientific computing was held at Zhejiang University. The purpose of that highly successful workshop concentrated on the introduction of VR and visualization technologies into the academic community of China. In parallel with the workshop, another advanced seminar on VR and visualization was held. Some foreign and domestic experts attending the international workshop were invited to give a series of lectures on directions and techniques in VR. The international workshop and the advanced seminar played an important role in promoting research activities on VR and visualization in China.

In 1996 VRST'96 (ACM Symposium on Virtual Reality Software and Technology) was held at the University of Hong Kong. This is the third VRST conference, and attracted a wide range of international attendees. It was a four-day conference with 4 half-day tutorials on the first day, followed by three days of technical presentations. The technical papers cover a wide range of subjects, varying from research topics to applications of VR. The authors presenting at the conference came from 13 different countries in Europe, Asia and North America.

In 1999 the International Workshop on Virtual Reality and Application (VR\&A), recognized as the successor of the first international workshop in 1995, was held in Zhejiang University. More than 100 attendees participated in the workshop (See Fig. 14). International experts came from U.S.A, Canada, Germany, Japan, Singapore, Hong Kong and Macao. Some VR experts from home and abroad were invited to deliver plenary presentations in the workshop. Detailed information for these talks is included in the accompanying CD-ROM of this special issue.

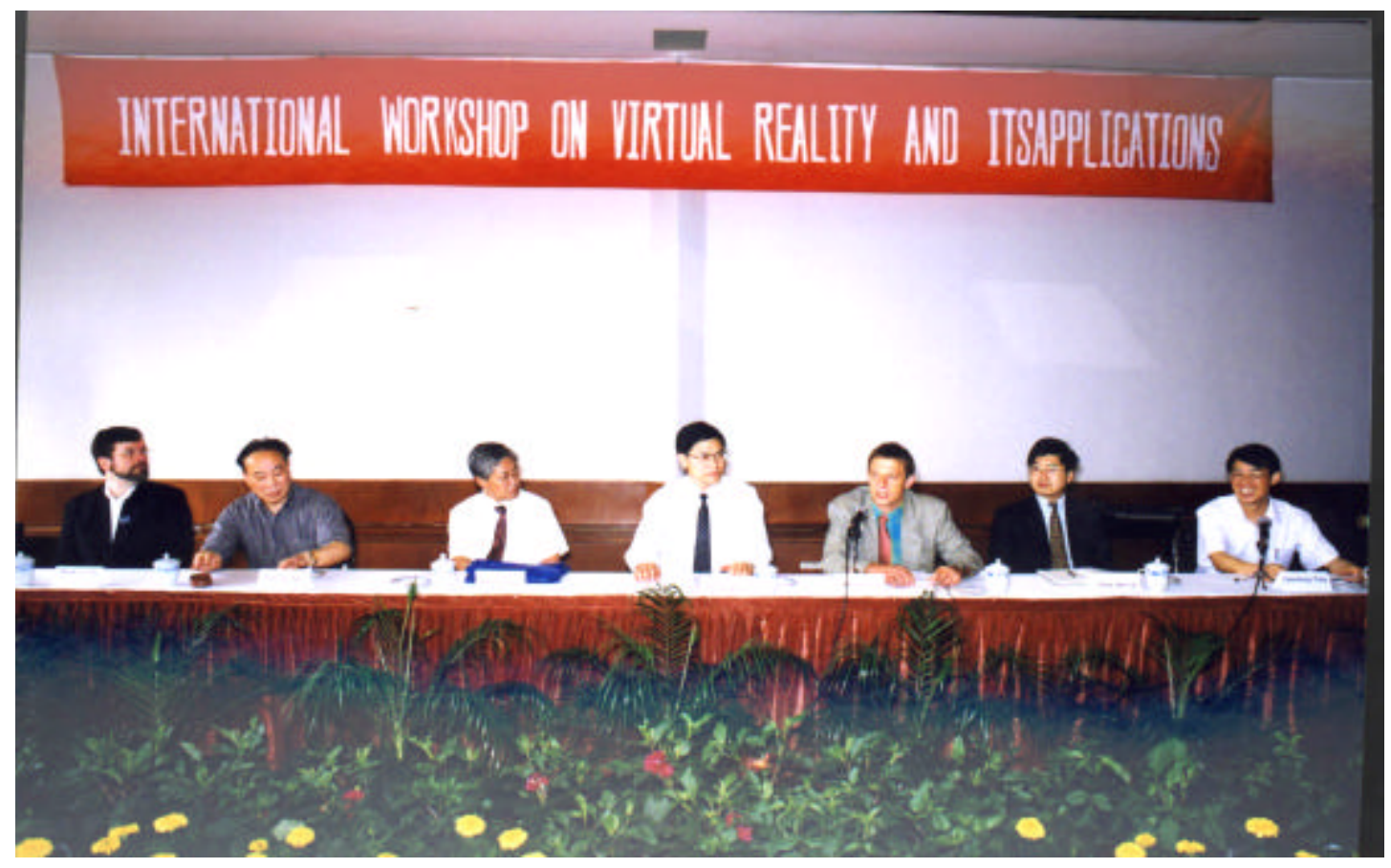

Fig. 14: Opening of the VR\&A Workshop 


\section{Summary of the Papers in the Printed Edition}

Our purpose in this special issue of the IJVR is to introduce the state of the art in VR research and development in China. Fortunately, many papers were submitted in many VR fields, including LoD/multi-resolution modeling, real-time rendering, image-based rendering, VR application (touring, medical treatment, teaching, training, visualization), distributed VR, virtual gesture recognition, behavior modeling, and computing model of VR.

We would like to thank all authors for their contributions. Eleven papers were selected to appear in the printed edition. In addition, ten papers are selected to appear on the CD-ROM edition. We shall now give a short summary of the papers on the printed edition. These papers may be divided into three groups: VR algorithms (IBMR, LoD), VR support/development environment (distributed VR, audio, sign language) and VR applications (medical applications and touring systems). Please refer to the CD-ROM for the papers that appear on CD-ROM only.

The first paper (by Tien-Tsin Wong et al.) is concerned with real-time display of arbitrarily complex scene. The authors discuss how two fundamental capabilities, navigation and illumination control, can be achieved in image-based virtual reality applications. To navigate through the imagebased scene correctly, they derive a triangle-based visibility-ordering algorithm from epipolar geometry.

The second paper (by Paul Bao et al.) presents a new view synthesis technique using the 2D discrete wavelet-based view morphing. The view morphing is completely based on pair-wise images without the camera calibration and the depth information of images.

The third paper (by Kun Zhou et al.) describes a new polygonal mesh simplification algorithm for colored or textured models based on vertex clustering. A more accurate error measuring method is introduced for vertex clustering. The authors also present an efficient interpolating method for LoD models.

The fourth paper (by Enhua $\mathrm{Wu}$ et al.) presents a novel method for real-time generation of progressive meshes. In their method, only the current mesh and readily accessible local information of each vertex are considered. Other properties such as vertex color and face texture are processed in the same way as geometry.

The fifth paper (by Yongjun Wang et al.) introduces a novel design method of distributed virtual environment development platform. A language model based on complex behavior and a computing model for virtual environments is proposed. They also describe a prototype system of distributed virtual environment application development system.

The sixth paper (by Qiong Zhang et al.) proposes a general-purpose virtual audio system. The authors discuss in detail the design and implementation techniques, including HRTF model based on neural network, the simulation of moving sound sources, the simulation of Doppler effects, and room acoustic simulation based on dynamic mechanisms.

The seventh paper (by Lin Xu et al.) is concerned with the generation and recognition of body language that can be used in VR systems. A graphical method is used to synthesize Chinese sign language, and a human-computer interaction system is implemented. 
The eighth paper (by Pheng-Ann Heng et al.) describes a virtual bronchoscopy system which simulates the real bronchoscopy operation. Doctor can diagnose a patient and plan for real bronchoscopic surgery using the system without risking patient's health.

The ninth paper (by Weidong Geng et al.) presents an image-based approach for virtual touring that use panoramas and picture-based animation to compose a virtual environment. Two typical touring behaviors, looking around and moving in one direction, are mimicked by players based on real-time image processing engine.

The tenth paper (by Bin Chan et al.) describes issues in designing a practical walkthrough system for indoor environments. The authors propose several new rendering speedup techniques implemented in their walkthrough system.

The eleventh paper (by Zigang Wang et al.) introduces a system for stereotactic neurosurgery planning. The system reconstructs and displays the 3D model of the interior structure of the patient's brain. Thus the surgeons can make the surgery planning on the model in the computer. This system also can be used to teach and train the new surgeons in the virtual reality environment.

\section{Conclusion}

VR is probably one of the most vigorously developing areas of computer graphics in China today. After several years of exploration, there have appeared many researchers and a number of VR companies. Both research and development in VR are robust, covering most branches of VR.

In such a short survey it is impossible to give a comprehensive picture of VR in China. We apologize in advance for any important missing regarding papers, achievements and products of VR in China. Finally, we hope the researchers in China can make promising achievements in the field of VR and its application in the future.

\section{Acknowledgement}

First of all, we would like to thank Prof. Richard Blade, the editor-in-chief of IJVR, for providing us the opportunity to introduce the VR research and development currently underway in China. We would like to express our sincere thanks to Ms. Lianhua Xiao (Computer Science Division, NSFC Committee), Prof. Danniel Yeung (Hong Kong Polytechnic University), Prof. Qunsheng Peng, Prof. Shuming Gao (Zhejiang University), Prof. Fuyan Zhang (Nanjing University) and Prof. Zesheng Tang (Tsinghua University) for their encouragement and support. Thanks also go to all the reviewers for their kind efforts and sincere comments.

Finally, we wish to thank Prof. Enhua Wu, Prof. Hua Li, Prof. Hujun Bao, Dr. Pheng-Ann Heng, Dr. Wenping Wang, Dr. Rynson Lau for their providing short introduction to the VR researches in their groups. In all, this special issue would not have been possible without generous support of the VR experts mentioned above.

This research work is partly supported by the Natural Science Foundation of China (Grant numbers 69773020 and 69823003). 


\section{REFERENCES}

[1] J.Y. Shi, Z.G. Pan "China: Computer Graphics is Fastest Developing Computer Application", ACM Computer Graphics, Vol.31,No.2, May. 1996.

[2] J.Y. Shi, Z.G. Pan (ed.) Special Issue on "Computer Graphics in China", Computers \& Graphics, Vol.21, No.3, 1996.

[3] J.Y. Shi, Z.G. Pan "China: Computer Graphics Education Available at Universities, Institutes and Training Centers", ACM Computer Graphics, Vol.31, No.3, August 1996.

[4] C.W. Wang, W. Gao, X.R. Wang, "The Theory, Implementation and Implementation of VR Technology", Tsinghua University Press, Beijing, 1996.

[5] X.H. Ma, Z.G. Pan, M.M. Zhang, et al. "The Automatic Generation of Multiple LoD and its Application", Progress in Natural Science, Vol.8 No.3, 1998, pp.346-353.

[6] C.Y. Cheng, Z.G. Pan, J.Y. Shi, "A Fast Algorithm For Generating Progressive Meshes", Journal of Image and Graphics, 3(11), 1998, pp.976-979.

[7] Z.L.Tao, Z.G. Pan, J.Y. Shi, “A Hierarchical Dynamic Simplification Method for Complex Environments", Journal of Image and Graphics, 3(12), 1998, pp.1032-1035.

[8] H.J. Bao, S. Fu, Q.S Peng, “Accelerated Walkthroughs of Complex Scenes Based on Visibility Culling and Image Based Rendering", Journal of Computer Science \& Technology, Vol.13, Supp., Dec, 1998, pp.10-16.

[9] Y.G. Wang, H.J. Bao, Q.S. Peng, "Accelerated Walkthroughs of Virtual Environments Based on Visibility Preprocessing and Simplification”, Computer Graphics Forum, Vol.17, No.3, 1998, pp.C187-194.

[10] H.J. Bao, L. Chen, J.G. Ying et al "Nonlinear view interpolation", Proceedings of Pacific Graphics'98, Singapore, 1998, pp.61-66.

[11] D. Xu, Z.G. Pan, J.Y. Shi, "Image-based Rendering Techniques for VR”, Journal of Image and Graphics, 3(12),1998,pp.1005-1010.

[12] D. Xu, "The Study and Implementation of IBMR in Wavelet Space", Ph.D. Dissertation, Zhejiang University, 1999.

[13] Z.G. Pan, J.Y. Shi, M.M. Zhang, "Distributed Graphics Support for Virtual Environment", Computers \& Graphics, 20(2), 1996.

[14] Z.G. Pan, M.M. Zhang, W.T. Zheng et al. "Time-critical Computing in Virtual Environment". Proceedings of CAD/Graphics'95, Wuhan, China, Oct. 1995,pp.1074-1079.

[15] Q. Zhang, J.Y. Shi, "Acoustic Simulation with Dynamic Mechanisms in VR", Journal of Computer Science \& Technology, 13(3), 1998, pp.285-288.

[16] Z.Gong, D.M. Lu and Y.H. Pan, "Dunhuang Art Cave Presentation and Preservation based on VE”, Technical Report, Zhejiang University, 1998.

[17] W.D. Geng, Y.H. Pan, M. Li, J. Yang, "Picture-based Virtual Touring”, IJVR, 4(4), 1999.

[18] J. Wang, "Real-time Visual Scene Management and Simulation System for DVE on PCs and Its Applications", Plenary Talk on the International Workshop on VR\&A, Hangzhou, China, June, 1999 [See the accompanying CD-ROM].

[19] J. Li, "Multi-resolution Modeling Techniques for 3D Complex Models", Ph.D Dissertation, Tsinghua University, 1998.

[20] T. Gui, "The Study and Implementation of the Real-time Dynamic Display of 3D Complex Models", Ph.D Dissertation, Tsinghua University, 1998.

[21] Z.G. Zhu, G.Y. Xu, X.Z. Lin, "Constructing 3D Real Full-view Model by Dithering Video 
Sequence", Journal of Image and Graphics, 3(9), 1998, pp.713-720.

[22] Y. J. Wang, The Research of Modeling Language and System Architecture of Distributed Virtual Environment and its Implementation, Ph.D Dissertation, University of Defense Technology, 1998.

[23] Y. Zheng, S.K. Li, C.J. Hu, et al., "A Cybernetics Based Behavior Model of Virtual Artifacts” Proceedings of CAD/Graphics'97, Shenzhen, China, 1997.

[24] L. Zhong, X.F. Hu, "Registering Algorithm for Overlapping Images", Journal of Image and Graphics, 3(5), 1998, pp367-370.

[25] G. Dang, S.Y. Jin, H.P. Hu, "A Reactive Multi-agent Simulation Environment Based on Object Web", Proceedings of National conference on Open Distributed and Parallel Processing, Heibei, China, 1999, pp141-148.

[26] Q.P. Zhao, X.K. Long, C.H. Xia et al. "DVENET: a Distributed Virtual Environment Network" Journal of Computer Research and Development, 1998, 35(12),pp.1064-1068.

[27] Z.Q. Wang, Q.P. Zhao, C.W. Wang, "The Representation and Processing of Physical Properties of the Objects in VE" Journal of Computer Research and Development, 1998, 35(2), pp.97-101.

[28] Z.Q. Wang, Q.P. Zhao, C.W. Wang, "OO-based Collision Detection and its Application in DVE", Chinese Journal of Computers, 1998,21(11), pp990-994 .

[29] T.L. Chung and W. Wang, "Quick Collision Detection of Polytopes in Virtual Environments" Proceedings of the 3rd ACM VRST'96, pp.125-132.

[30] C.H. Poon and W. Wang (1999), “ Occlusion Culling Using Minimum Occluder Set and Opactiy Map”, Proceeding of Information Visualization, ‘99, July, 1999, London.

[31] B. Chan, H.M. Tse, and W. Wang, " Design of a Walkthrough System for Indoor Environments from Floor Plans”. Proceedings of Information Visualization '98, pp.50-57.

[32] P. A. Heng, P. F. Fung, T. T. Wong, et al. "Interactive Navigation and Bronchial Tube Tracking in Virtual Bronchoscopy", in the Proceedings of Medicine Meet Virtual Reality:7, IOS Press, San Francisco, January 1999, pp.130-133.

[33] H. Sun, X. Yuan, G. Baciu, Y. Gu, "Direct Virtual-hand Interface in Robot Assembly Programming", The Journal of Visual Languages and Computing, special issue on User Interfaces and Interaction in Virtual Reality, Vol. 10, 1999, pp. 55-68.

[34] T. T. Wong, P. A. Heng, S. H. Or et al., "Illumination of Image-based Objects", Journal of Visualization and Computer Animation, Vol. 9, 1998, pp.113-127.

[35] D. Xu \& P. Bao, Wavelet-based Progressive View Morphing, Visual Communications and Image Processing'99, SPIE, 3653, 1999 ,pp.613-619.

[36] P. Bao, \& D. Xu, Complex Wavelet-based Image Mosaics using Edge-Preserving Visual Perception Modeling, Computers and Graphics, 23(3), June ,1999.

[37] Z.G. Pan, K. Zhou, C.Y. Cheng, et al. "Level of Detail and Multi-resolution Modeling Techniques for Virtual Design and Prototyping”, Proceeding of 1999 Industrial Virtual Reality Symposium, Chicago, U.S.A, Nov. 1999.

[38] R. Lau, M. Green, D. To, et al., "Real-Time Continuous Multi-Resolution Method for Models of Arbitrary Topology," Presence, MIT Press, 7(1), Feb. 1998, pp.22-35.

[39] J. Chim, R. Lau, A. Si, et al., "Multi-Resolution Model Transmission in Distributed Virtual Environments," Proceedings of ACM VRST, Nov. 1998, pp.25-34.

[40] J. Chim, M. Green, R. Lau, et al.”On Caching and Pre-fetching of Virtual Objects in Distributed VE," Proceedings of ACM Multimedia, Sept. 1998, pp.171-180.

[41] W. Wang, Y.Q. Jing, S.H. Dong, "A PC-based platform for VR development-PCVRS", Proceedings of CAD/Graphics'97, Shenzhen, China, 1997.

[42] F.P. Xu, B. Xu, H.J. Ding et al. "Virtual Driving Training Simulator for Automobile", IJVR,4(4)[CD-ROM edition],1999.

[43] H.B. Li, D.M. Zhang, S.F. Chen, "Research on the design methodology and computing models 
for VR environment", Chinese Journal of Computers, 22(3), 1998.

[44] J.B. Zhang, T. Yang, J.Z. Sheng, "Continuous multi-resolution modeling based on vertex decimation", Journal of Image and Graphics, 4(5), 1999, pp.395-399.

[45] H. Zhao, Y.Y. Yu, Y.H. Liang, et al. "RTG: A Powerful Toolkit for Real-Time 3D Graphics", IJVR,4(4)[CD-ROM edition],1999.

[46] E.H. Wu, "Virtual Reconstruction of Church St. Paulo from its Ruins" Plenary Talk on the International Workshop on VR\&A, Hangzhou, China, June, 1999 [See the accompanying CDROM].

[47] X.Y. Ma, "Research on Walkthrough and Texture Generation in Virtual Reality", Master Thesis, Institute of Computing Technology, 1999.

[48] X.J. Yuan, J.C. Ren, "Fractional-level zooming of Multi-resolution images" Proceeding of the 5th International Young Computer Scientists Conference, Nanjing, China, 1999.

[49] Y.Q. Xu, C. Su, D.X. Qi, et al. "Physically Based Simulation of Water", Computers \& Graphics. 1997, 21(3),pp.277-280.

[50] X.H. Liu, E.H. Wu, "Rendering Acceleration of Terrain Models Based on Image Space Criterion", Chinese Journal of Software, 9(7), July 1998, pp.481-486.

[51] Y. Cai, P-A. Heng, E.H. Wu, et al. "QuickShow: an Image-based Virtual Reality Prototype System", Journal of Computer Science and Technology, 13(5), Science Press of China \& Allerton Press of USA, 1998.

[52] H.J. Li, E.H. Wu, "A New Representation of Panoramic View for an Indoor Virtual Environment”, in ACM Symposium on Virtual Reality Science \& Technology(VRST'98), Nov. 1998.

\section{WEB URLS:}

Zhejiang University: http://www.zju.edu.cn

State Key Lab of CAD\&CG, Zhejiang University: http://cad.zju.edu.cn

Intelligent CAD Lab, Department of Computer Science and Engineering, Zhejiang University: mailto:Panyh@sun.zju.edu.cn

National Lab of Human Factors, Zhejiang University: mailto:jwang@mail.hz.zj.cn

Department of Computer Science and Technology, Tsinghua University: http://www.tsinghua.edu.cn

National University of Defence Technology: http://www.nudt.edu.cn

Beijing University of Aeronautics and Astronautics: http://www.buaa.edu.cn

Computer Graphics Group, The University of Hong Kong: http://www.csis.hku.hk/GraphicsGroup/main.htm

VR, Visualization and Imaging Research Centre, The Chinese University of Hong Kong: http://www.cse.cuhk.edu.hk/ pheng

VR Lab, Hong Kong Polytechnic University: mailto:cszgpan@comp.polyu.edu.hk

VR Group of the City University of Hong Kong: http://www.cs.cityu.edu.hk/ rynson/

CAD Lab, Institute of Computing Technology, Chinese Academy of Sciences: mailto:lihua@ns.ict.ac.cn

Lab of Computer Science, Institute of Software, Chinese Academy of Sciences: mailto:weh@ox18.ios.ac.cn

Lab of Engineering Science for Complex Systems, Institute of Automation at the Chinese Academy of Sciences: mailto:mxj7106@hotmail.com

Sunstep Inc, Beijing: http://www.sunstep.com.cn

Shanghai 3D Technology Co., Ltd, Shanghai, China: mailto:dddchina@guomao.sh.cn

Voyager Corp., Shongshan, Guangdong Province: mailto:image@public.zhongshan.gd.cn

Real-time Graphics Inc., China: mailto:rgb@public.bta.net.cn 


\section{BIOGRAPHIES}

Zhigeng Pan is a professor at the State Key Lab of CAD\&CG, Zhejiang University, a member of the IS\&T, a senior member of the China Image and Graphics Association. He received M.S. degree from Nanjing University and Ph.D. degree from Zhejiang University, all in computer graphics. He is a content editor for the International Journal of Virtual Reality, and on the Editorial Board of Journal of Image and Graphics. He has published more than 60 papers and is the author and co-author of three books. He acted as one of the Guest Editors for a Special Issue of Computers \& Graphics. His current research interests include VR, distributed graphics and multimedia. Currently, he is visiting the Department of Computing, the Hong Kong Polytechnic University as a Research Fellow, responsible for directing research in the fields of VR and CG.

Jiaoying Shi is a professor of the Department of Computer Science and Engineering at Zhejiang University, China. He is the Director of Academic Committee of State Key Lab of CAD\&CG, the Deputy Chairman of China Image and Graphics Association, the Deputy Chairman of China CAD and Graphics Society under China Computer Federation. He is a member of SIGGRAPH Education Committee, and on the Editorial Board of international journal Computers \& Graphics. Since 1990 his work is concentrated on visualization and virtual environment. He has published more than 100 papers and three books.

\section{Contact information:}

Prof. Jiaoying Shi,

State Key Lab of CAD\&CG, Zhejiang University, Hangzhou, 310027, China

Phone: +86-571-7951045

Fax: $+86-571-7951780$

Email: mailto:jyshi@cad.zju.edu.cn

Qin Lu received her B.Sc. degree from E.E. Department, Beijing Normal University and her M.Sc. and Ph.D. degrees from the Computer Science Department, the University of Illinois at UrbanaChampaign. She is now an associate professor at the Department of Computing, Hong Kong Polytechnic University. Her research interests include distributed computing, information retrieval and standardization, multimedia and multilingual computing, VR and Chinese Computing.

\section{Contact information:}

Dr. Qin Lu VR Lab, Department of Computing, The Hong Kong Polytechnic University, HK, China

Phone: $+852-27667247$

Fax: $+852-27740842$

Email: mailto:csluqin@comp.polyu.edu.hk 